Classification

Physics Abstracts

42.30.Yc - 81.70.Fy - 81.05.Rm

\title{
The Holodisc Distance Transform and its Applications in Image Analysis
}

\author{
Éric Pirard \\ Université de Liège, MICA, 45 avenue des Tilleuls, 4000 Liège, Belgium
}

\begin{abstract}
Résumé. - Les fonctions distances sont essentielles en analyse d'images. Non seulement elles permettent d'obtenir rapidement l'ensemble des érodés ou dilatés d'un objet, mais elles ont des propriétés topographiques remarquables. Pour des raisons avant tout algorithmiques, ce sont les fonctions distances en métrique carrée ou hexagonale qui sont à la base du plus grand nombre de développements. Nous montrons ici qu'en adoptant une fonction distance holodisque, il est possible d'accéder à de puissants outils d'analyse ou de segmentation morphologique en bénéficiant de toute la précision d'une métrique Euclidienne.
\end{abstract}

\begin{abstract}
Distance transforms are essential tools in image analysis for obtaining the complete spectrum of eroded or dilated sets from a given object. Moreover, their remarkable topographical properties have led to numerous developments in the field of shape segmentation and analysis. Mostly for algorithmical reasons, these operators have been derived from square or hexagonal distance transforms. In this paper, we show how the holodisc distance function makes it possible to access even more powerful tools while taking advantage of a truly Euclidean metric.
\end{abstract}

\section{Introduction}

Optical images obtained from black and white or color video cameras are rarely quantitatively analysed as such except for luminance or chrominance studies where the intensities represent a physical quantity of interest to the application. In most other situations optical images are taken to enable the segmentation of the various objects (phases) present in a scene (material). By segmentation one means any procedure resulting in a univocal classification of pixels or groups of pixels into a limited number of bins.

Geometric measurements in image analysis proceed from binary images and are either pixelbased or neighbourhood-based. As an example of this:

- area measurements are pixel-based: it suffices to count the number of object-pixels in an image without considering their position;

- perimeter measurements are neighbourhood-based: a simplistic polygonal perimeter estimation (Poly_4) is given by counting the number of object-pixels that have at least one background-pixel in their elementary neighbourhood $(3 \times 3$ square $)$. 
Table I. - Perimetric estimation for a disc and a rectangle using a set of images taken at increments of $7.5^{\circ}$ with respect to the digital grid. The true values are 274.13 for the disc and 390 for the rectangle. Poly_4 gives the number of border pixels in the square grid, Poly-8 is the length of an 8-connexity polygonal line linking border pixels. $P_{-} C r \_3$ is the Crofton estimator based on the three major intercepts of the pseudo-hexagonal grid and $P_{-} \overline{C r}_{-} 4$ is the Crofton estimator based on the four intercepts of the octagonal grid $(0, \pi / 4, \pi / 2$ and $3 \pi / 4)$.

\begin{tabular}{|l|l|l|l|l|}
\hline & Mean & Min & Max & Std.Dev \\
\hline \hline Poly_4 & 346.8 & 344 & 351 & 2.41 \\
\hline Poly_8 & 288.8 & 286.6 & 292.1 & 1.80 \\
\hline P_Cr_3 & 292.1 & 281.0 & 300.1 & 6.42 \\
\hline P_Cr_4 & 276.0 & 273.8 & 279.1 & 1.71 \\
\hline
\end{tabular}

\begin{tabular}{|l|l|l|l|l|}
\hline & Mean & Min & Max & Std.Dev \\
\hline \hline Poly_4 & 487.3 & 392 & 543 & 56.82 \\
\hline Poly_8 & 408.4 & 391.0 & 421.3 & 10.65 \\
\hline P_Cr_3 & 406.7 & 373.3 & 421.2 & 17.55 \\
\hline P_Cr_4 & 389.1 & 372.6 & 401.3 & 10.08 \\
\hline
\end{tabular}

Fundamentally, pixel-based and neighbourhood-based procedures are like looking at a scene with eye-flaps, the global context is missing. A simple illustration of this is given by the problem of unbiased perimeter estimation. Using the most classical neighbourhood-based algorithms for analysing a disc and a rectangle one gets the results of Table I. These results are truly experimental in the sense that they have been obtained by putting a geometrical figure drawn on a white paper under the camera and by rotating it every $7.5^{\circ}$ around the optical axis. The scaling used is such that we got an average of 5980 pixels for the disc and 6043 pixels for the rectangle. Clearly, Crofton's formula [1], performs much better than any other method. But, since this formula has been designed for isotropic figures the standard deviation is quite high for the rectangle and one must accept a possible error of up to $5 \%$ for this particular shape. The octagonal approximation (Poly_8) is best suited for polygonal shapes with sides along a $n \pi / 4$ direction but overestimates the perimeter for any other orientation. In other words, if one wants to improve the perimeter estimation of such shapes, one must be able to recognize the existence or absence of long range arrangements in the set of border pixels (either as pieces of arcs or segments).

For solving such a problem, one could imagine looking at very large neighbourhoods rather than restricted local ones, but this is most often totally inefficient. Pattern recognition techniques based on identification of high curvatures deal with similar problems. The and Chin [2] present a good review of some recent literature in this field.

The basic idea behind global perception in binary images is the geodesic propagation [3]. A propagation means that information is transferred from one pixel to its neighbours, while geodesic means this operation is restricted to the set of connected pixels. The most familiar illustration of geodesic propagation is the labelling procedure used for denumbering individual blobs in an image (Fig. 1). But a propagation function might as well carry other information and in particular a dynamic recording of the length of a path. By counting the minimum number of steps from a source point $\mathrm{A}$ to a destination point $\mathrm{B}$, a so-called geodesic distance is recorded.

\section{Distance Mappings Using Different Metrics}

The most widespread definition of a distance transform (DT) of an object (X) is a mapping in which each object-pixel takes a value equal to the minimum number of steps required to reach the background $\left(\mathrm{X}^{\mathrm{C}}\right)$. Background-pixels can also be mapped as negative distances but more usually they are left at zero by the DT. Referring to Figure 2, a distance mapping algorithm can be defined by imagining that all border pixels act as source points for a geodesic propagation. The DT result for a given point is then given by the minimum distance front reaching this point. 


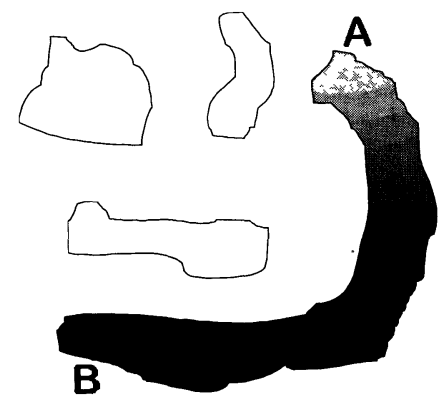

Fig. 1. - The labelling operator is a geodesic propagation. A color label introduced in A floods the whole object and reaches extremity B.

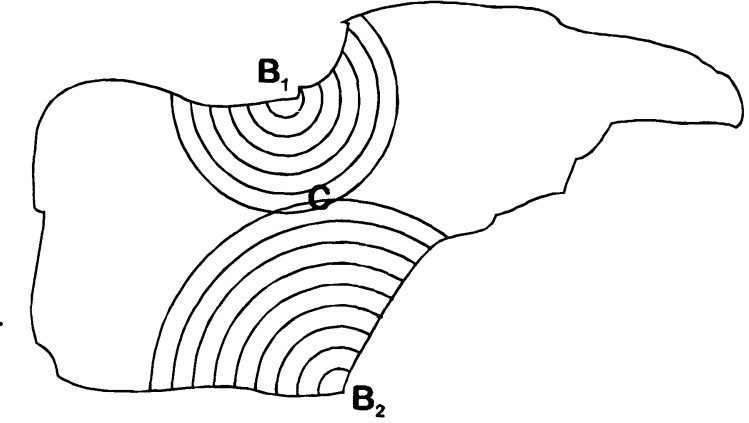

Fig. 2.

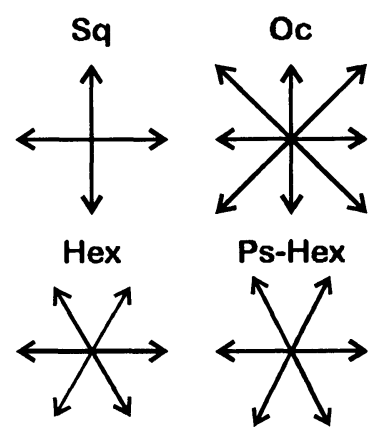

Fig. 3.

Fig. 2. - Geodesic propagation waves issued by all possible border points $B_{i}$ reach point $\mathrm{C}$. A distance transform will only retain the value of the smallest distance.

Fig. 3. - Some elementary neighbourhoods based on square rasters: square $\left(a_{0}=a_{90}\right)$; octagonal $\left(a_{45}=a_{135}=1.414 a_{0}\right)$; pseudo-hexagonal $\left(a_{63}=a_{117}=1.118 a_{0}\right)$ and on triangular rasters: hexago$\operatorname{nal}\left(a_{0}=a_{60}=a_{120}\right)$.

Up to now the distance has been expressed as a number of steps in order to give a unique definition. There are however as many possible DTs as there are definitions for steps. The notion of step being directly related to the kind of metric one associates with the grid. Mainly for algorithmical reasons, discrete metrics are largely dominant in the field of image analysis. These are either square and octagonal metrics on square rasters, or hexagonal and dodecagonal metrics on triangular rasters (Fig. 3).

Because the metric is the main link between digital images and the real world, it is strongly recommended to calibrate the real dimensions of the vertices $\left(a_{0}, a_{90}, \ldots\right)$ obtained with any imaging device. Some video acquisition boards still grab a full video frame into a $512 \times 512$ raster, thereby inducing a rectangular metric $\left(a_{90} \cong 0.667 a_{0}\right)$ instead of a square one. In using algorithms based on hexagonal neighbourhoods one must be aware that these are pseudo-hexagonal, unless the sampling frequency of the frame grabber has been adjusted. 


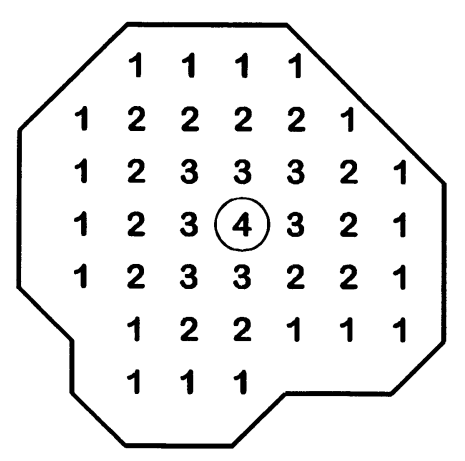

Fig. 4.

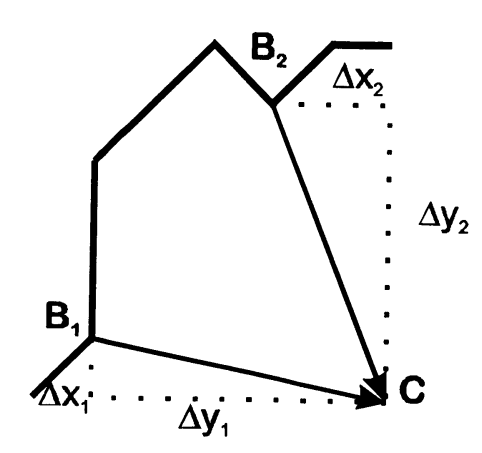

Fig. 5.

Fig. 4. - Close view of a distance transform using a square metric. The ultimate eroded set is indicated by the circle. Hence, the radius of the maximum inscribed square is 4 .

Fig. 5. - The Euclidean distance transform is obtained by retaining the vector of minimum norm reaching C. Only the NW quadrant (considered during the first scan) is illustrated here.

The discrete DTs mentioned above are easily implemented using two image scans [4]:

$$
\begin{array}{lll}
\text { forward scan : } & \left\{\begin{array}{l}
P_{0}=0 \Rightarrow P_{0}^{*}=0 \\
P_{0}=1 \Rightarrow P_{0}^{*}=\operatorname{Min}\left\{a_{i}+P_{i}\right\}
\end{array}\right. \\
\text { backward scan : } & \left.\left\{\begin{array}{l}
P_{0}=0 \Rightarrow P_{0}^{*}=0 \\
P_{0} \neq 0 \Rightarrow P_{0}^{*}=\operatorname{Min}\left\{P_{0}, a_{i}+P_{i}\right\}
\end{array} \quad \forall i \in\right] 180^{\circ}, 360^{\circ}\right]
\end{array}
$$

with: $P_{0}$ the value of the current pixel (centre of the neighbourhood), $P_{0}^{*}$ the value of $P_{0}$ in the output image, $a_{i}$ the length of the step in direction $i, P_{i}$ the neighbour of $P_{0}$ in direction $i$.

Except the true square (Fig. 4) and hexagonal distances, the DT use non-integer $a_{i}$ values and require memory-consuming floating point operations. An approximation of an Euclidean distance using integer steps has been proposed by Borgefors [5]. This distance is often abusively called Euclidean by several authors.

A true Euclidean DT supposes to be able to propagate vectors instead of values. This means that one must record $\Delta x$ and $\Delta y$ images for each quadrant and compute the minimum norm for each vector reaching a point of the object (Fig. 5). Such an algorithm requires four image scan [6].

Considering the fact that an Euclidean distance mapping on a discrete grid only yields a very limited subset of Euclidean distance values (e.g. distance 2.78 will never exist on a square grid), the holodisc distance transform was recently introduced [7]. The holodisc distance $\left(d_{\mathrm{O}}\right)$ between two points $\left(x_{1}, y_{1}\right)$ and $\left(x_{2}, y_{2}\right)$ is such that:

$$
d_{\mathrm{O}}-1<\sqrt{\left(x_{1}-x_{2}\right)^{2}+\left(y_{1}-y_{2}\right)^{2}} \leq d_{\mathrm{O}}
$$

with $d_{\mathrm{O}} \in \mathrm{N}_{0}$.

Practically, the same algorithm is kept to compute the Euclidean distance, but values are rounded up to the integer. With a holodisc DT (from oגos: integer and $\delta \iota \sigma \chi \omega$ : disc), pixels take the value of the largest disc with integer radius that can be centred on them without hitting a background pixel. To be precise, this is the case if the border-pixels (those belonging to the inner eight-connexity contour) are considered to be at a distance of zero. 


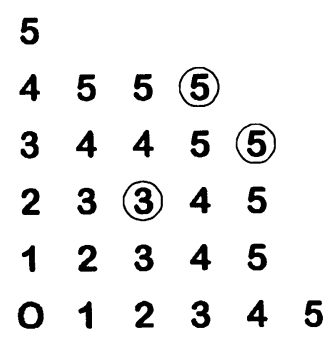

Fig. 6 .

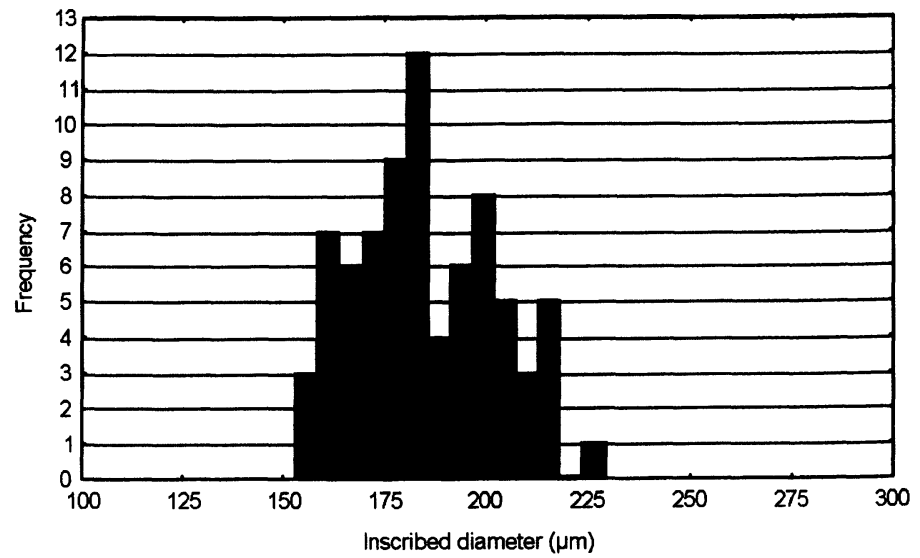

Fig. 7.

Fig. 6. - Holodisc distance wavefronts propagating from the lower left pixel. Eight-connexity of the isolevels is always guaranteed and locally some extra thickness points (circled) are added.

Fig. 7. - Histogram of the maximum inscribed holodiscs diameters $\left(2 \lambda_{\mathrm{E}}\right)$ obtained on a batch of $200 \mu \mathrm{m}$ diamonds.

\section{On the Usefulness of Distance Transforms}

At first glance, a distance mapping closely resembles a topographic map with its summits, valleys and crest lines. This analogy is very helpful in order to grasp some useful features of distance transforms.

Having a closer look at the contour levels of the map, one could notice that levels of discrete DTs exactly parallel each other while the isolevels of a Euclidean DT appear disconnected. Interestingly, a holodisc DT guarantees the eight-connexity of the contour levels but locally adds some extra thickness (Fig. 6). From a practical point of view, thresholding a distance map at level $n$ gives the image of the object after an erosion of size $n$. For discrete metrics, this could also have been obtained by $n$ iterations of elementary erosions, but for Euclidean metrics, the DT is the most efficient way to get the $n$-eroded set. By duality, the correct Euclidean dilation is deduced from a DT applied to the background.

GranUlometry. - Beside filtering, the main use of the erosion operation in image analysis is in granulometry. The basic notion therefore are the "ultimate eroded sets (UE)" which are nothing else than the summits of the distance map obtained by marking all pixels at level $n$ having no connected neighbour at level $n+1$ (Fig. 4). The value of an UE in a distance map is equivalent to the size of the maximum inscribed polygon (for a holodisc DT this is the radius $\lambda_{\mathrm{E}}$ of the maximum inscribed holodisc). If one is interested in the granulometric analysis of powder particles, it is easy to show that the measure of the maximum inscribed holodisc diameter $\left(2 \lambda_{\mathrm{E}}\right)$ is perfectly correlated with the results of a sieving operation if the hidden dimension (thickness) belongs to ]0,2 $\lambda_{\mathrm{E}}$ (Fig. 7). More precisely, because sieves are made of square meshes, the true granulometry by sieving will lie somewhere between the results of the square DT (for infinitely thin particles) and the holodisc DT (for particles as thick as $2 \lambda_{\mathrm{E}}$ ). In practice, because particles are irregular and often contain several disconnected UEs, it is recommended to retain only the highest value of UE within each individual particle. 


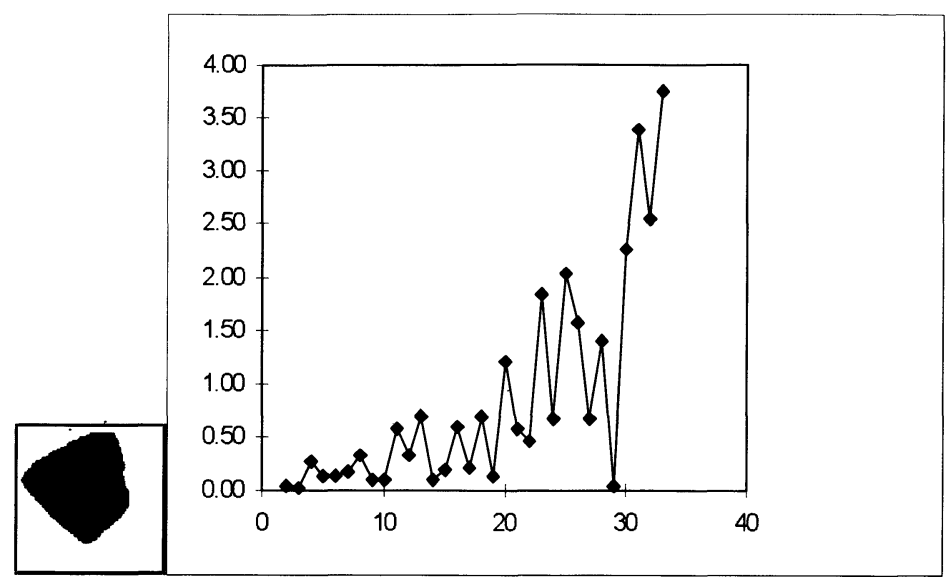

Fig. 8. - The holodisc opening spectrum is given by the derivative of the area measurement against the size of the opening.

Generalized Granulometry and Morphometry. - Matheron [8] showed that the opening operation fulfills the fundamental axioms of a granulometric analysis. A complete opening spectrum for an object can be obtained from iterations of erosion/dilation cycles of increasing size. More efficiently, the same opening spectrum has been derived directly from the hexagonal distance map [9] and from the square distance map [10]. This last algorithm can be extended to any metric giving homothetic discrete structuring elements. The calyptre descriptor [7] makes it possible to compute directly from a holodisc DT the centre and radius of each maximum inscribed holodisc associated to a contour point. From this, it is straigthforward to compute the holodisc opening spectrum (Fig. 8) giving the area fraction lost after each opening with a holodisc of increasing radius.

The opening spectrum (sometimes also called pattern spectrum) is claimed to be very useful in shape analysis or in image segmentation, but very few practical applications have been published [11-13]. Obviously, analyses relying on a non-isotropic metric suffer from a severe lack of robustness and the use of a truly Euclidean metric like the holodisc DT is the only way to minimize the influence of rotation and scaling of the digital grid on the scattering of results. In a previous paper [14], a roughness parameter $\left(W_{\mathrm{V}}\right)$ making use of all the maximum inscribed holodisc radii given by the calyptre was introduced. Figure 9 shows how this parameter behaves when a set of images are taken from a sand grain with the camera being rotated by increments of $7.5^{\circ}$.

Coding AND Shape HieraRCHY. - Unlike spline decriptors based on points of high curvature, the calyptre is a vectorial descriptor preserving the full geometrical information of the contour. It is possible therefore to develop shape coding capabilities by inducing a progressive loss of geometrical information. As an example of this, a closing (dilation followed by erosion) of the radii signal $(\Lambda)$ of the calyptre can be defined using:

$$
\begin{array}{ll}
\text { dilation : } & D(\Lambda)=\left\{\lambda_{i}^{*}: \max \left(\lambda_{i-1}, \lambda_{i}, \lambda_{i+1}\right)\right\} \\
\text { erosion : } & E(\Lambda)=\left\{\lambda_{i}^{*}: \min \left(\lambda_{i-1}, \lambda_{i}, \lambda_{i+1}\right)\right\}
\end{array}
$$

with $\lambda_{i}$ the radius of the maximum inscribed holodisc associated to the $i$ th contour pixel. 


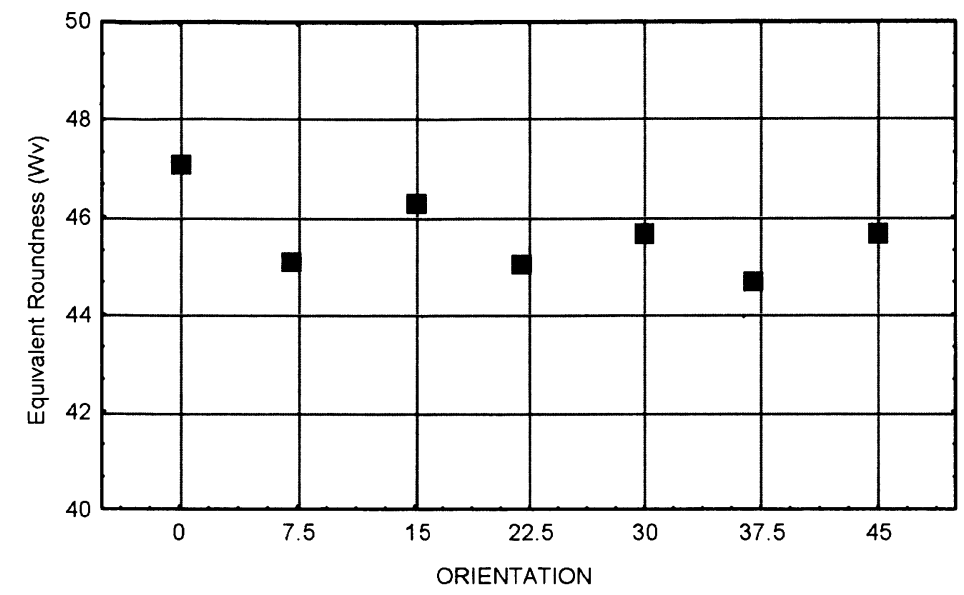

Fig. 9. - Evolution of the equivalent roundness $\left(W_{\mathrm{V}}\right)$ of a sand grain (12.000 pixels) with rotation of the digitization grid. Experimental values of $W_{\mathrm{v}}$ for natural sand particles are between $30 \%$ and $90 \%$. Measures have been obtained after a recommended triple closing of the radii signal of the calyptre.
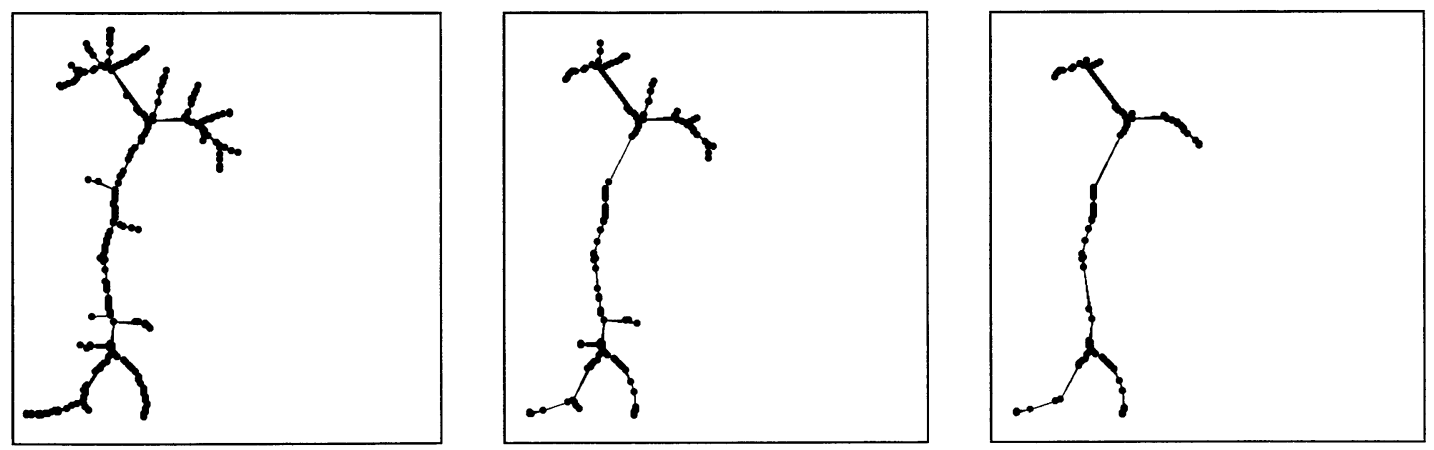

Fig. 10. - Representation of a hierarchy of holodisc centres preserved by increased closings $(3 \times, 6 \times, 9 \times)$ of the calyptre radii signal. The holodisc centres are joined by straight lines to facilitate visual comparison with skeleton hierarchies.

If one excepts the case of shapes with multiple UEs, such a closing will remove from the calyptre those maximum inscribed holodiscs that justify only one contour pixel located in between contour pixels justified by larger holodiscs. If a stronger closing is performed by taking into account more neighbours, than the number of holodisc is further decreased and the process progressively converges towards the ultimate opened set (Fig. 10). Closing of the radii signal with increasing neighbourhoods develops a calyptre hierarchy that is a very powerful alternative to time-consuming skeleton hierarchies [15].

\section{Conclusion}

Discrete distance transforms like the square and octagonal ones have been preferred in the past to the Euclidean distance transform because of their simpler topography. Skeletons and granulometries are among the most interesting tools that have been computed from such distance mappings. 
It is shown here that the uprounding of the Euclidean distance (called holodisc distance) allows to benefit from a simpler topography of the distance mapping without loosing the exactness of the Euclidean metric. Other developments based on the holodisc distance like watersheds and voronoï polygons are being considered.

\section{References}

[1] Serra J., Image analysis and Mathematical Morphology (Academic Press, London, 1982).

[2] Teh C. and Chin R., On the detection of dominant points on digital curves, IEEE Trans. Patt. Anal. Mach. Intell. 2 (1989) 859-872.

[3] Lantuéjoul C. and Beucher S., On the use of the geodesic metric in image analysis, J. Microsc. 121 (1981) 39-49.

[4] Rosenfeld A. and Pfaltz J., Sequential operations in digital picture processing, J. Assoc. Comp. Mach. 13 (1966) 471-494.

[5] Borgefors G., Distance Transformations in digital images, Comp. Vision Graph. Image Proc. 34 (1986) 344-371.

[6] Danielsson P.E., Euclidean Distance Mapping, Comp. Graph Image Process 14 (1980) 227-248.

[7] Pirard E. and Nivart J.F., New descriptor for skeletons of planar shapes: the calypter, Proc. SPIE/IST, Non Linear Image Processing V, E. Dougherty, Ed., 2180 (1994) 248-259.

[8] Matheron G., Eléments pour une théorie des milieux poreux (Masson, Paris, 1967).

[9] Laÿ B., Recursive algorithms in Mathematical Morphology, Acta Stereol. 6 (1987) 691-696.

[10] Vincent L., Fast opening functions and morphological granulometries, Proc. SPIE Image algebra and morphological image processing V, E. Dougherty, Ed., 2300 (1994).

[11] Frossard E., Caractérisation pétrographique et propriétés mécaniques des sables, Thèse de Doctorat de l'École des Mines, Paris (1978).

[12] Chermant J.L., Coster M. and Gougeon G., Shape analysis in $\mathbb{R}^{2}$ using mathematical morphology, $J$. Microsc. 145 (1987) 143-157.

[13] Vincent L. and Dougherty E., Morphological segmentation for textures and particles, in "Digital Image Processing: fundamentals and applications”, E. Dougherty, Ed. (Marcel-Dekker, New York, 1993).

[14] Pirard E., Shape processing and analysis using the calypter, J. Microsc. 175 (1994) 214-221.

[15] Ogniewicz R. and Kübler O., Hierarchic Voronoï Skeletons, Patt. Recog. 28 (1995) 343-359. 